E3S Web of Conferences 5, 01001 (2015)

DOI: $10.1051 /$ e3sconf/ 20150501001

(C) Owned by the authors, published by EDP Sciences, 2015

\title{
Analysis of the sweeped actuator line method
}

\author{
Jörn Nathan ${ }^{1, a}$, Christian Masson ${ }^{1}$, Louis Dufresne ${ }^{1}$, and Matthew Churchfield ${ }^{2}$ \\ ${ }^{1}$ École de Technologie Supérieure, Montréal, QC, Canada \\ ${ }^{2}$ National Renewable Energy Laboratory, Golden, CO, USA
}

\begin{abstract}
The actuator line method made it possible to describe the near wake of a wind turbine more accurately than with the actuator disk method. Whereas the actuator line generates the helicoidal vortex system shed from the tip blades, the actuator disk method sheds a vortex sheet from the edge of the rotor plane. But with the actuator line come also temporal and spatial constraints, such as the need for a much smaller time step than with actuator disk. While the latter one only has to obey the Courant-Friedrichs-Lewy condition, the former one is also restricted by the grid resolution and the rotor tip-speed. Additionally the spatial resolution has to be finer for the actuator line than with the actuator disk, for well resolving the tip vortices. Therefore this work is dedicated to examining a method in between of actuator line and actuator disk, which is able to model the transient behaviour, such as the rotating blades, but which also relaxes the temporal constraint. Therefore a larger time-step is used and the blade forces are swept over a certain area. The main focus of this article is on the aspect of the blade tip vortex generation in comparison with the standard actuator line and actuator disk.
\end{abstract}

\section{Introduction}

When the actuator line method (ALM) was introduced by Sørensen and Shen [1], it became possible to model the effects of the rotor in the near wake more accurately since the generation of the helicoidal vortex structure shed from the blade tips is taken into account. The disadvantage of this approach is the need for a very small time-step compared to the widely used actuator disk method (ADM) [2]. For the ALM the blade effects are represented by line forces as shown in Figure 1(a). The time-step has to be smaller than the time a point on the blade tip needs to pass through one cell. Therefore it is given by $\Delta t_{A L M}=\Delta x / U_{\text {tip }}$ with the uniform cell length $\Delta x$ in the rotor region and the tip-speed $U_{t i p}$. With a full rotation of the line forces and their azimuthal averaging, the ADM is obtained as depicted in Figure 1(c). Therefore the time-step for this method is given by the Courant-Friedrichs-Lewy (CFL) condition, hence $\Delta t_{A D M}=\Delta x / U_{\infty}$ with the ambient velocity $U_{\infty}$. With a typical tip speed ratio in the range of 6 to 8 and together with the fact that the ALM requires a 4 to 5 times smaller grid spacing, the time-steps for the ALM end up to be 20 to 40 times smaller than the ones for the ADM. Also the spatial resolution has to be fine enough for representing the tip vortices. If the grid is too coarse, the generated vorticity will be shed in form of a vortex sheet as obtained by the ADM.

Despite these temporal and spatial restrictions, the ALM is widely applied for the description of the near wake [3] [4], but also for the dynamics of whole wind farms as shown by Churchfield et al. [5].

\footnotetext{
ae-mail: joern.nathan.1@ens.etsmtl.ca
} 
This work focuses on relaxing the temporal constraint by examining a hybrid model between ALM and ADM, the sweeped actuator line method (SALM) as discussed by Storey [6] and Sanderse [7]. By fanning out the line forces as shown in Figure 1(b), the time-step only needs to respect the CFL condition:

$$
\begin{aligned}
\Delta t_{S A L M} & =\Delta t_{A D M} \\
& =\Delta x / U_{\infty} \\
& =\left(U_{t i p} / U_{\infty}\right)\left(\Delta x / U_{t i p}\right) \\
& =\lambda \Delta t_{A L M}
\end{aligned}
$$

and therefore the time-step for the SALM can be a multiple of the ALM, depending on the tip-speed ratio. The time-step of the ALM is depending on the spatial discretization in the vicinity of the rotor. A very fine mesh leads to a very small time-step, hence the swept area of the SALM at each time-step can also become very small even if it is running with a multiple of the time-step of the ALM.

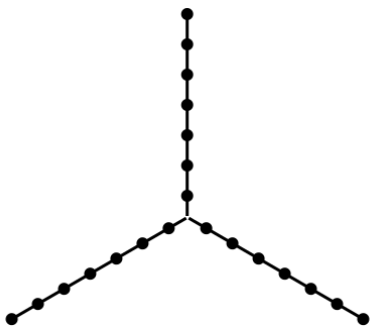

(a) ALM

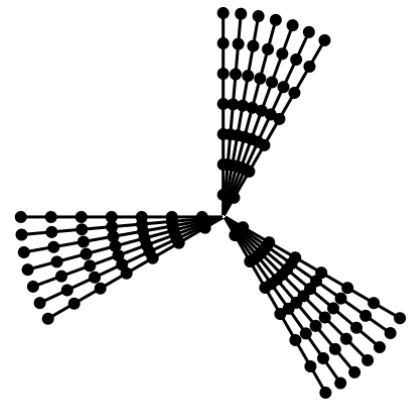

(b) Sweeped ALM with $\Delta t=7 \Delta t_{A L M}$

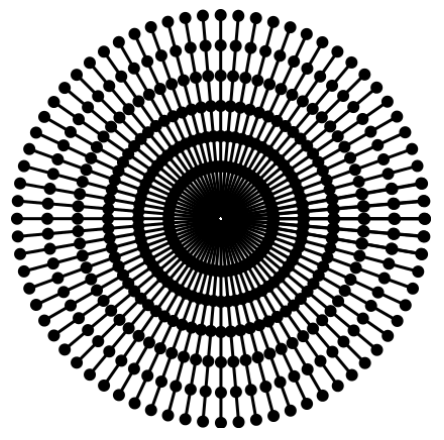

(c) ADM

Figure 1. Actuator point distribution in the rotor plane for the three different models after the first time-step.

camissa negra

\section{Method}

Based on the existing implementation of an ALM according to Sørensen and Shen [1] in the SOWFA ${ }^{1}$ project the SALM and ADM are implemented in OpenFOAM ${ }^{2}$, an open-source $\mathrm{C}++$ framework for computational fluid dynamics. Using object-oriented programming an abstract class of a turbine model is created whose child classes are the ALM, sweeped ALM and the ADM. All three methods are based on inserting the rotor effects as forces in the momentum equations, and therefore this generalization facilitates the specific adaptation for each method. In the second part of this section, the numerical setup of the examined case are presented.

\subsection{Rotor modeling}

While the ALM implemented in the SOWFA project calculates a line of forces for each blade at each time-step as shown in Figure 1(a), the sweeping ALM divides the time-step in sub time-steps as shown

\footnotetext{
${ }^{1}$ NWTC Design Codes (SOWFA by Matt Churchfield and Sang Lee). http://wind.nrel.gov/designcodes/simulators/SOWFA/. Last modified 14-March-2012; accessed 14-March-2012.

${ }^{2}$ Copyright (C)2004-2013 OpenCFD Ltd (ESI Group).
} 
in Figure 1(b). An ADM can be obtained by calculating the forces by a full rotation at each time-step as shown in Figure 1(c). As the incoming velocity remains constant and irrotational for the simulated cases a converged body force can be used for the ADM after a certain time in order to speed up the simulation. This only holds for constant, non-turbulent inflow cases.

In order to avoid an unphysical oscillation across the rotor plane when introducing a punctual force as source term in the momentum equations, the forces associated with the blades are distributed in a Gaussian manner with a deviation of $\epsilon=2 \Delta x$ as suggested by Troldborg [10]. These distributed forces are then inserted as a source term in the momentum equations.

\subsection{Case setup}

In order to compare the different actuator methods, three cases are set up. The examined turbine is the NREL 5 MW reference turbine as described by Jonkman et al. [9] with an ambient velocity of $U_{\infty}=8 \mathrm{~m} / \mathrm{s}$ and a tip-speed ratio of $\lambda \approx 7$.

The domain extends $5 D$ in the streamwise and in each lateral direction with the rotor placed at $2 D$ after the inlet. These dimensions are chosen in order to reduce simulation runtime and when comparing the results to larger domains, no significant differences are found in the results of the near wake up to $1 D$ behind the rotor. In the rotor and near wake region a uniform cubic cell distribution with the length $D / 64$ is used. This region extends $2 D$ in streamwise and $1.5 D$ in each lateral direction whereas the region begins at $x / D=-0.5$. The cells outside of this uniform region are stretched towards the domain boundaries whereas the maximum aspect ratio of around 15 occurs at the outlet.

The cases with ALM and ADM are set up with their respective time-steps and the one with SALM with $\Delta t_{S A L M}=\Delta t_{A D M}=\lambda \Delta t_{A L M}$. The computational overhead due to the fanning of the forces leads to an increase of the execution time of each simulation time-step as shown in Table 1. But at the same time fewer time-steps are necessary for SALM and ADM compared to ALM, which outweighs the increased execution time per time-step. The total execution time for the SALM for this case is about one fifth of the one of the ALM. The ADM as implemented in this case leads only to a slightly higher total execution time than the SALM. It is expected to be much higher for turbulent inflow cases.

\begin{tabular}{llll}
\hline & ALM & SALM & ADM \\
\hline execution time per time-step & 1.00 & 1.45 & 1.61 \\
total execution time & 1.00 & 0.20 & 0.23 \\
\hline
\end{tabular}

Table 1. Execution time for different actuator models.

For describing the flow large-eddy simulations (LES) are used and the closure is achieved by the classic Smagorinsky model with a constant model coefficient $C_{S}$ of 0.167 . It is important to mention, that there is no turbulence from the incoming flow and therefore the only turbulent structures result from the breakdown of the vortex structure in the wake.

For the velocity a Dirichlet condition with $\mathbf{U}=\left(U_{\infty}, 0,0\right)$ is imposed at the inlet and a Neumann condtion with $\partial \mathbf{U} / \partial n=0$ at the lateral boundaries and the outlet. For the pressure also a zero gradient with $\partial p / \partial n=0$ is applied on all boundaries .

The divergence term $\operatorname{div}(\mathrm{phi}, \mathrm{U}$ ) is discretized by using the filteredLinear scheme. This is done in order to avoid the occurence of spurious oscillations in the flow field, which are generated when the flow exhibits high velocity gradients and the discretization is done by pure central differencing. This can also be avoided by applying a hybrid scheme which blends between central differencing and a scheme with upwind character as done by Troldborg [10] and Martínez-Tossas [11]. This reduction of accuracy guarantees more physical results. The filteredLinear scheme acts similarly 


\begin{tabular}{llllll}
\hline & & ALM & SALM & ADM & Ref. \\
\hline torque & {$[\mathrm{MNm}]$} & 2.04 & 2.21 & 1.99 & 1.95 \\
diff & {$[\%]$} & 4.61 & 13.3 & 2.0 & - \\
\hline
\end{tabular}

Table 2. Torque values for different actuator models.

to a total variation diminishing (TVD) scheme, as it also applies a flux limiter based on the gradients of the current cell and its neighbours, but at the same time it does not obey the rules postulated by [8] and therefore it can not be categorized as TVD.

For the remaining spatial terms the central differencing scheme (linear) is chosen. For the temporal discretization the Crank-Nicolson method is used.

A transient PISO algorithm is used for solving the Navier-Stokes equations with one pressure corrector step. When varying the number of pressure corrector steps, it could be seen that the influence on the results in the near wake is negligible. In order to reduce simulation runtime only a single pressure corrector step is applied. For solving the matrices, a generalised geometric-algebraic multigrid (GAMG) is used for the pressure and a preconditioned bi-conjugated gradient (PBiCG) for the velocity.

\section{Results}

When looking at the generated torque of the turbine in Table 2, it can be seen that ALM and ADM are fairly close to the reference value [9] compared to the SALM, which is off by more than $13 \%$. This is due to the approach how the velocities for the calculation of the blade forces are sampled. For the ALM the velocities are sampled at the last position of the blade, so inside of the vortex generated by the inserted force. For the SALM the velocities should probably be sampled in the middle of the last fan which is not yet implemented during this work. As the main goal for this article is the analysis of the vortex system generated by the method, obtaining the right torque is left for future work. In Figure 2 the isosurfaces of the body force magnitude visualize how the forces are spread for the different actuator methods. While the ALM exhibits a tubular distribution for each blade, the forces for the SALM are more fanned out and for the ADM the forces are swept over the whole rotor plane.

When looking at the vorticity structure in Figure 3 and Figure 4, it can be observed that the ALM creates a helicoidal vortex system which is generated by the circulation around the blade and shed at the blade tips into the downstream direction. As the vortices shed by the ALM are more distinct when comparing to SALM in Figure 5(a) and Figure 5(b), the resulting structure remains intact further downstream than with the SALM. While the distinct vortices merge into a vortex sheet in the near wake of the SALM, the ADM directly sheds a vortex sheet as shown in Figure 3 and Figure 5(c). 


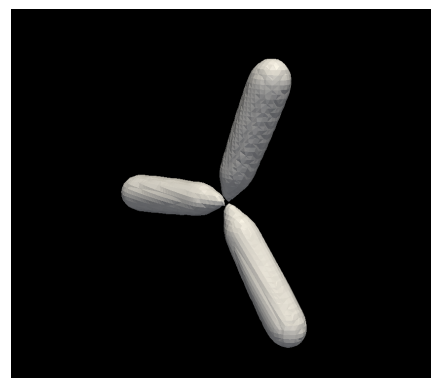

(a) ALM

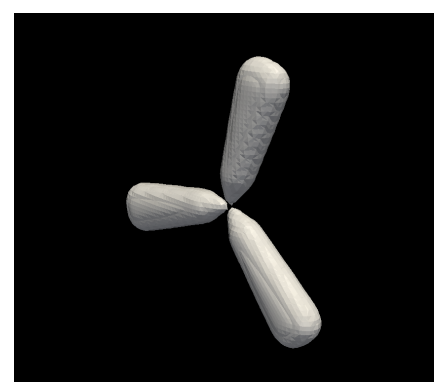

(b) SALM

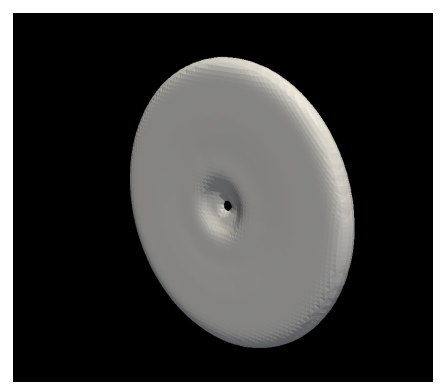

(c) ADM

Figure 2. Isosurfaces of body force magnitude for each rotor model.

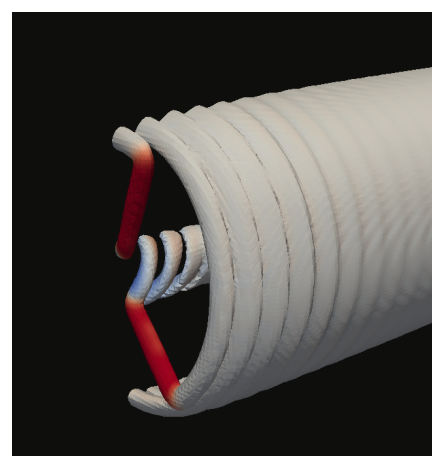

(a) ALM

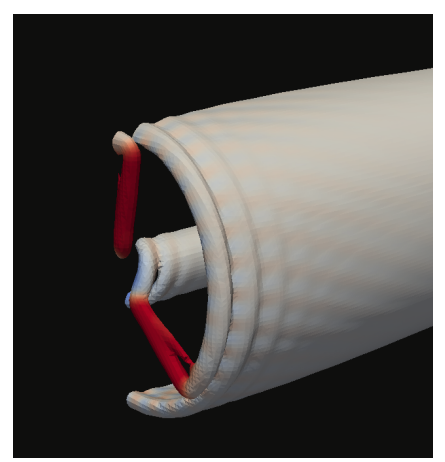

(b) SALM

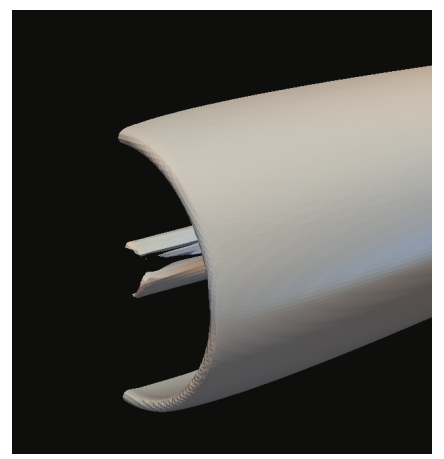

(c) ADM

Figure 3. Isosurfaces of vorticity magnitude coloured by radial vorticity for each rotor model.

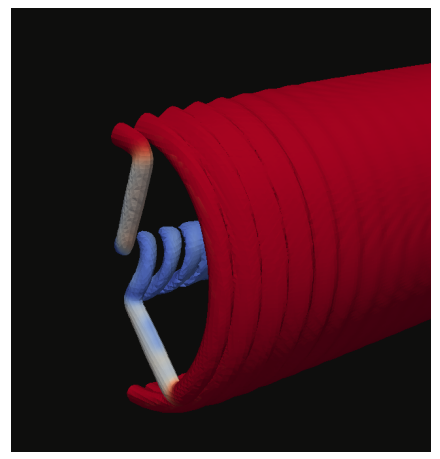

(a) ALM

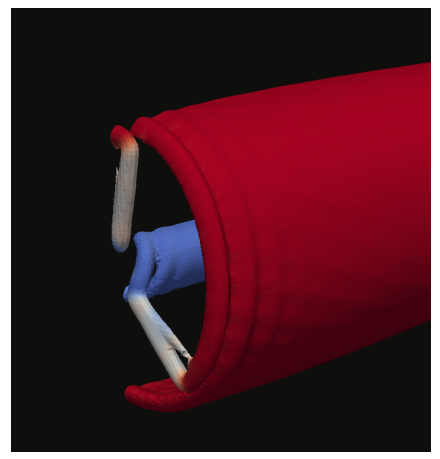

(b) SALM

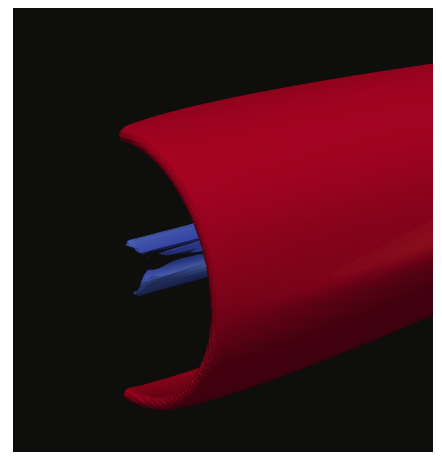

(c) $\mathrm{ADM}$

Figure 4. Isosurfaces of vorticity magnitude coloured by azimuthal vorticity for each rotor model. 
In order to take a closer look on the vortex generation, the vorticity is decomposed in its radial and azimuthal component with the radial direction from the blade root to its tip and the azimuthal direction in the rotating sense. As OpenFOAM uses cartesian coordinates as reference system, the vorticites are obtained by the following transformation

$$
\begin{aligned}
\omega_{\Phi} & =\boldsymbol{\omega} \cdot \hat{\boldsymbol{r}} \\
\omega_{r} & =\boldsymbol{\omega} \cdot \hat{\boldsymbol{\Phi}}
\end{aligned}
$$

with the unit vectors

$$
\begin{aligned}
& \hat{\boldsymbol{r}}=\hat{z} \cos \left(\arcsin \left(\frac{y}{\sqrt{z^{2}+y^{2}}}\right)\right)+\hat{\boldsymbol{y}}\left(\frac{y}{\sqrt{z^{2}+y^{2}}}\right) \\
& \hat{\boldsymbol{\Phi}}=-\hat{z}\left(\frac{y}{\sqrt{z^{2}+y^{2}}}\right)+\hat{\boldsymbol{y}} \cos \left(\arcsin \left(\frac{y}{\sqrt{z^{2}+y^{2}}}\right)\right)
\end{aligned}
$$

It can be seen that the circulation around the blades in Figure 3(a) is shed as vorticity into the wake in Figure 4(a), whereas the ADM sheds a vortex sheet with the same magnitude as in the cases of the ALM and SALM.

When looking at the velocites in the near wake in Figure 6 it can be seen that there is only small difference in downstream direction which can be attributed to the fact that the flow exhibits no initial turbulence and therefore the wake recovery takes place very slowly. Due to this absence of dissipative effects and with the scope only on the near wake, the discrete nature of each blade section can clearly be noticed in the transversal profile. The turbulent shear stress in Figure 7 shows a distinct peak for the ALM in the shear layer of the wake, which smears out a bit for the SALM and is absent for the ADM. As the examined case exhibits no turbulence, the velocity fluctuations stem solely from the periodic passage of the blade tip vortices. Hence this stress indicates the presence and strength of the tip vortices, which are less distinct for the SALM compared to the ALM. This can be seen as the trade-off for gaining a faster simulation by fanning out the forces.

\section{Conclusion}

The SALM is implemented within the SOWFA project and compared to the ALM and the ADM. Similarly to the ALM it sheds a vortex system consisting of three helicoidail vortices shed from the blade tips, but as they are less distinct this structure breaks up earlier downstream and merges into a vortex sheet as with the ADM. When looking at the shear stresses it could be seen, that it also exhibits a distinct peak of $\left\langle u^{\prime} w^{\prime}\right\rangle$ within the shear layer of the wake although it is more smeared out than with the ALM. In future work it would be interesting to examine also turbulent inflow and change the interpolation between different blade sections for removing the abrupt changes in the downstream transversal velocity profiles. Another improvement of the method would also be the change of how velocities are sampled for the calculation of the blade forces, as it has a huge impact on the calculation of the torque. 


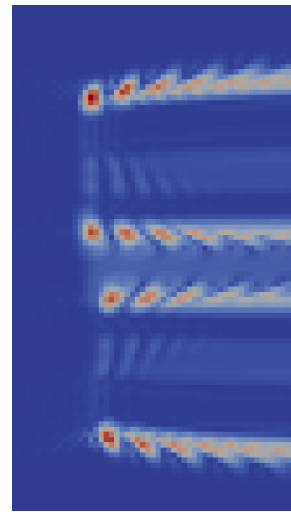

(a) ALM

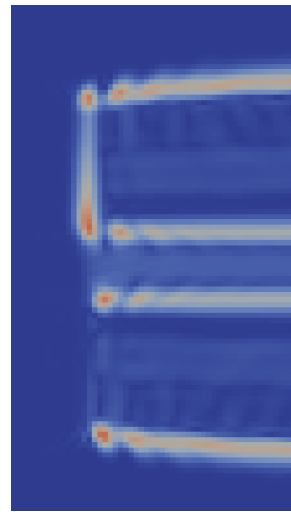

(b) SALM

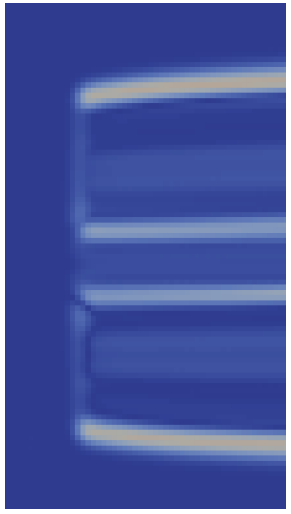

(c) $\mathrm{ADM}$

Figure 5. Cutting plane of vorticity magnitude in streamwise and radial direction for each rotor model.

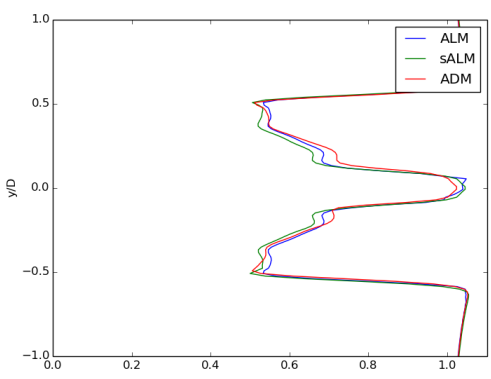

(a) $x / D=0.5$

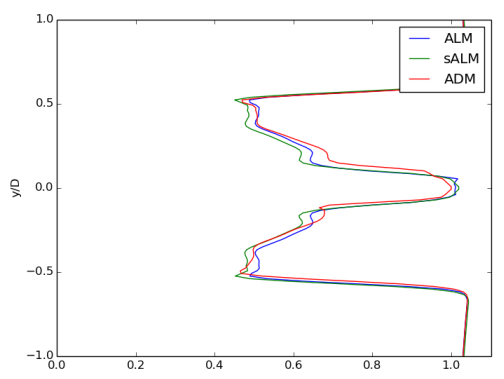

(b) $x / D=1.0$

Figure 6. Normalized mean transversal streamwise velocity profile $\langle U\rangle / U_{\infty}$ at different locations in the near wake.

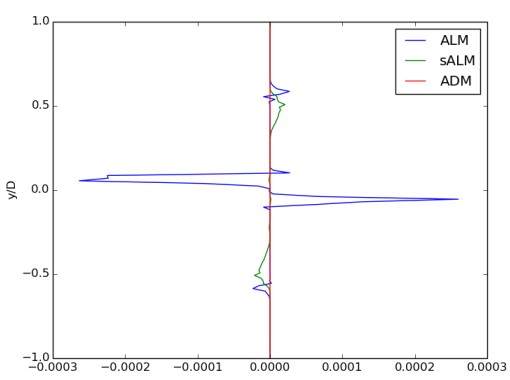

(a) $x / D=0.5$

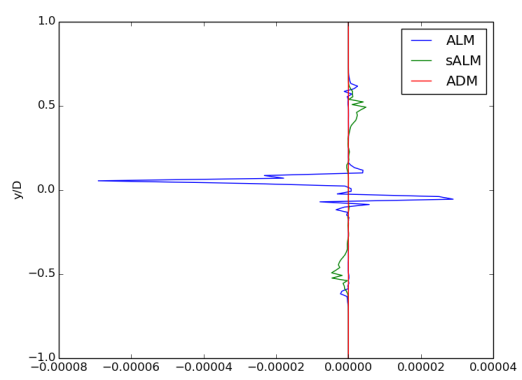

(b) $x / D=1.0$

Figure 7. Normalized mean transversal shear stress profile $\left\langle u^{\prime} w^{\prime}\right\rangle / U_{\infty}^{2}$ at different locations in the near wake. 


\section{References}

[1] N.N. Sørensen, W.Z. Shen, Numerical Modeling of Wind Turbine Wakes, Journal of Fluids Engineering, 124, 393-399 (2002)

[2] Yu-Ting Wu, F. Porté-Agel, Large-Eddy Simulation of Wind-Turbine Wakes: Evaluation of Turbine Parametrisations, Boundary-Layer Meteorology, 138, 345-366 (2011)

[3] S. Ivanell, R. Mikkelsen, J.N. Sørensen, D. Henningson, Stability analysis of the tip vortices of a wind turbine, Wind Energy, 13, 705-715 (2010)

[4] P.-Å. Krogstad, J.A. Lund, An experimental and numerical study of the performance of a model turbine, Wind Energy, 15, 443-457 (2012)

[5] M. Churchfield, S. Lee, P. Moriarty, L. Martinez, S. Leonardi, G. Vijayakumar, J. Brasseur, A Large-Eddy Simulation of Wind-Plant Aerodynamics, 50th AIAA Aerospace Sciences Meeting including the New Horizons Forum and Aerospace Exposition (2012)

[6] R. Storey, S. Norris, J. Cater, An actuator sector method for efficient transient wind turbine simulation, Wind Energy, 18, 699-711 (2015)

[7] B. Sanderse, B. Koren, Immersed Actuator Methods, Conference: The Science of Making Torque from Wind, Oldenburg, 2012

[8] A. Harten, High Resolution Schemes for Hyperbolic Conservation Laws, Journal of Computational Physics, 135, 260-278 (1997)

[9] J. Jonkman, S. Butterfield, W. Musial, G. Scott, Definition of a 5-MW Reference Wind Turbine for Offshore System Development, Technical Report, NREL (2009)

[10] N. Troldborg, Actuator Line Modeling of Wind Turbine Wakes, $\mathrm{PhD}$ thesis, Technical University of Denmark, Copenhagen, 2008

[11] L. Martínez-Tossas, M.J. Churchfield, S. Leonardi, Large eddy simulations of the flow past wind turbines: actuator line and disk modeling, Wind Energy, 18, 1047-1060 (2015) 